\title{
Case Report: Surgical Treatment of Two Asymptomatic Myxomas at Two Atypical Locations
}

\author{
Lana Maričić MD, PhD, ${ }^{1,2}$ Grgur Dulić MD, PhD, ${ }^{1,2}$ Sandra Makarović MD, PhD, ${ }^{1,2}$ \\ Vlatka Periša MD, ${ }^{1,2}$ Livija Sušić, $M^{3}$ \\ ${ }^{1}$ University Hospital Osijek, Osijek, Croatia; ${ }^{2}$ Faculty of Medicine Osijek, University J.J. Strossmayera, Osijek, Croatia; \\ ${ }^{3}$ Health Centre Osijek; Osijek, Croatia
}

\section{ABSTRACT}

Atypical presentation of myxomas in the two cases described here arise from the fact that both patients were asymptomatic and both showed unexpected echocardiographic findings. Asymptomatic presentation is very rare, and occurs in only about $10 \%$ of individuals. Atrial myxomas discovered on incidental echocardiography is also a rare phenomenon, as seen in our cases. Early diagnosis and timely surgical treatment allow these patients to live a completely asymptomatic life.

\section{CASE PRESENTATION I}

A 72 year old man was presented in our echocardiography laboratory to assess left ventricular systolic function in preparation for hematological treatment of immunochemotherapy for previously diagnosed non Hodgkin B mantle cell lymphoma. Transthoracic echocardiography (TTE) verified a mobile cardiac mass in the left atrium, which protruded into the left ventricle during diastole. Transesophageal echocardiography (TEE) confirmed the existence of a cardiac mass, which pushes the posterior mitral leaflet, and consequently causes a mild mitral regurgitation (Figure 1). The patient underwent cardiac surgery, and cardiac masses were removed. Pathological examination confirmed a giant cardiac myxoma of $4 \times 4 \times 2 \mathrm{~cm}$ (Figure 2). Histologically, the mass was built of myxoid structures without significant atypia, and some bleeding and elements of fibrin were found in the mass. The postoperative course was uncomplicated. The control TTE showed a non-dilated left atrium, without significant mitral regurgitation (Figure 3 ).

\section{CASE PRESENTATION 2}

A 74 year old woman had a long history of hypertension and diabetes mellitus, and was being treated by a psychiatrist for depressive syndrome. She was asymptomatic, and specifically denied chest pain, dyspnea, palpitation, and syncope. TTE identified a mass of $1.6 \times 1.9 \mathrm{~cm}$ which adhered to the

Received Fanuary 9, 2017; accepted February 25, 2017.

Correspondence: Lana Maričić, MD, PbD, Department of Cardiovascular Diseases, University Hospital Osijek, 7.Huttlera 431000 Osijek, Croatia; 38531511724 fax: +38531512169 (e-mail:dr.lmaricic@gmail.com). posterior mitral leaflet, was entirely located in the left ventricle, and didn't protrude into the left atrium (Figure 4). Color Doppler showed mild mitral regurgitation, and moderate tricuspid regurgitation with consequent indirect elevated pressure in the right ventricle (RVSP $44 \mathrm{mmHg}$ ). The

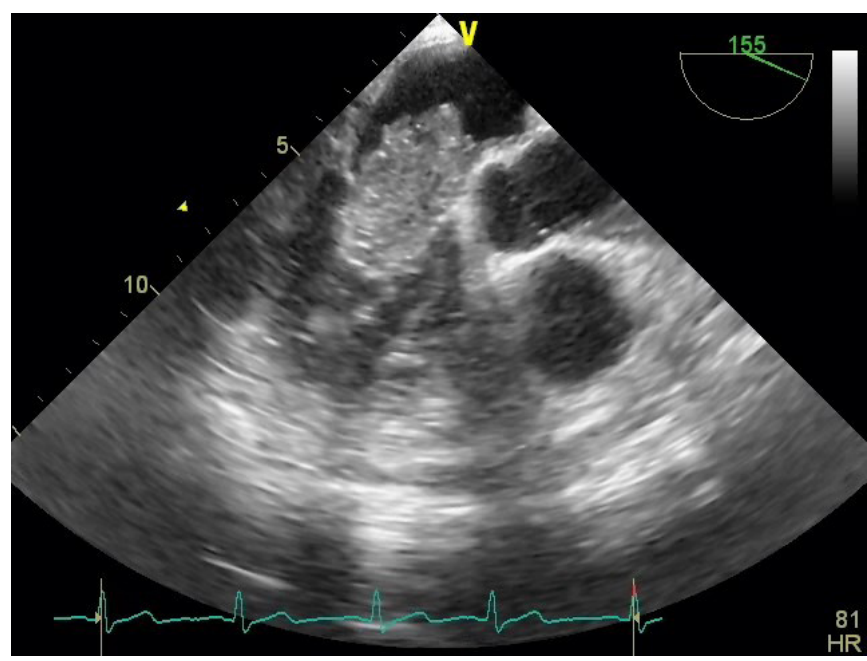

Figure 1. TEE, transgastric view. Cardiac mass in the left atrium which protrudes into the left ventricle depending on the phase of the cardiac cycle.

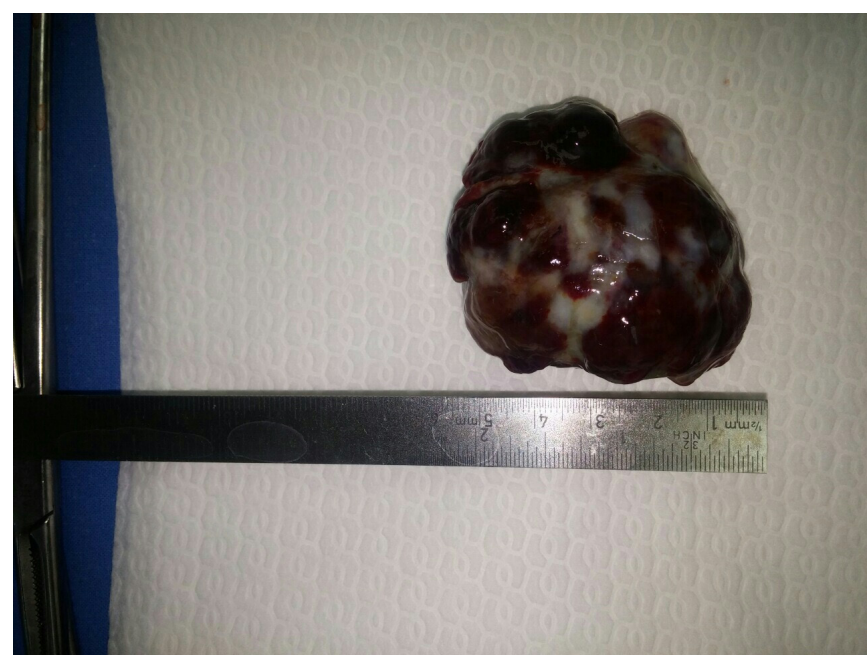

Figure 2. Macroscopic intraoperative view of cardiac mass. 


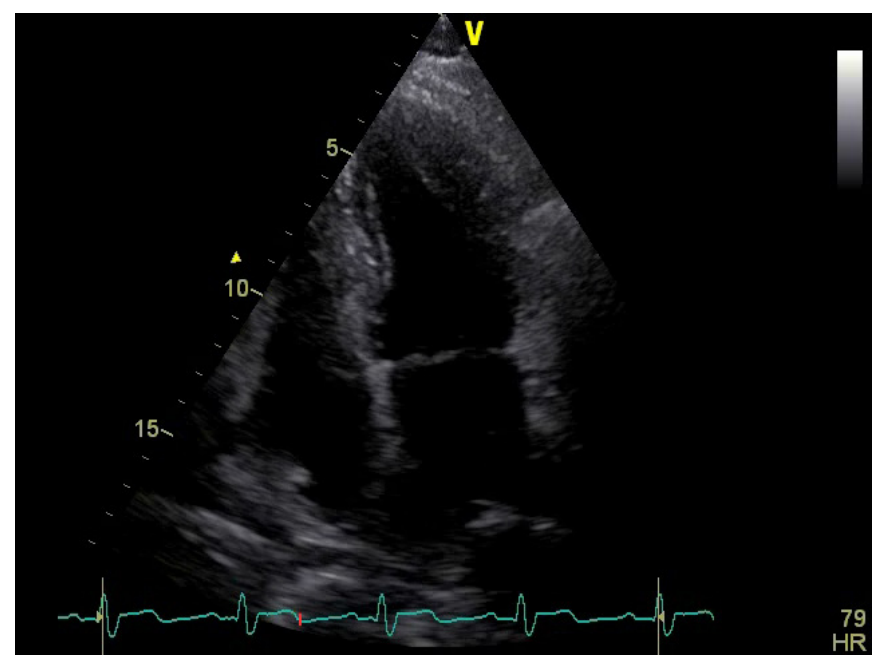

Figure 3. TTE, Control echocardiography after cardiac surgery. The apical four chamber view. The transmitral color doppler signal shows a non significant mitral regurgitation.

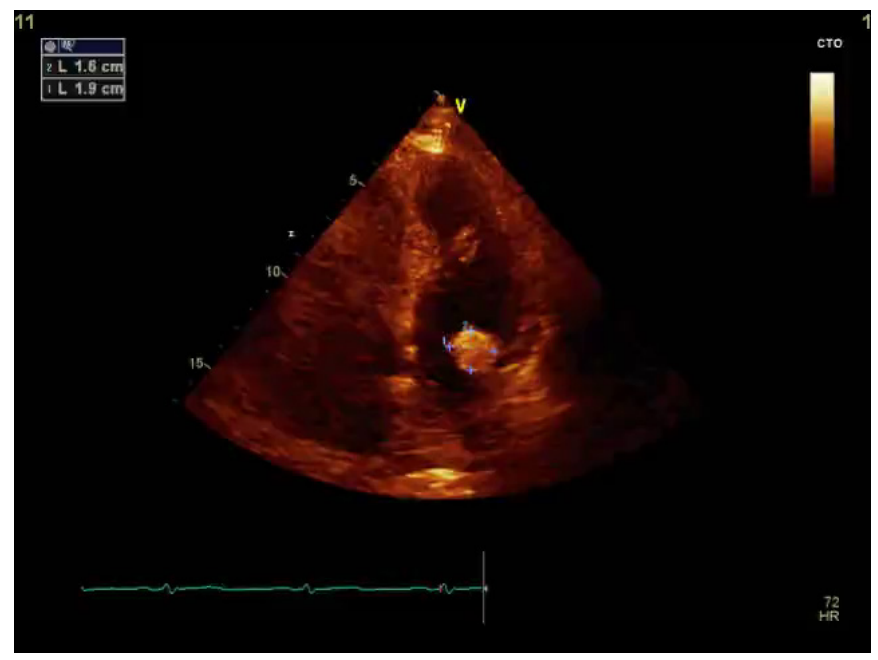

Figure 4. TTE, the apical four chamber view. Solitary hyperechoic intracardiac mass attached to the posterior mitral leaflet, measuring 1.6 $\times 1.9 \mathrm{~cm}$.

contractility of both ventricles was preserved. TEE confirmed the above findings. Coronarography showed normal findings on the epicardial vessels. The patient underwent cardiac surgey, and cardiac masses were removed and replaced with a biological mitral valve. Pathologic examination confirmed a cardiac mass which was dominantly built of myxoid structures. The postoperative course was uncomplicated. On the control TTE, adequate positions of the biological mitral valve were visualized, with an appropriate flow rate (Figure 5).

\section{DISCUSSION}

Approximately $80 \%$ of individuals present with one component of the triad, yet up to $10 \%$ may be asymptomatic [Nath 2011]. Cardiac myxomas usually develop within the

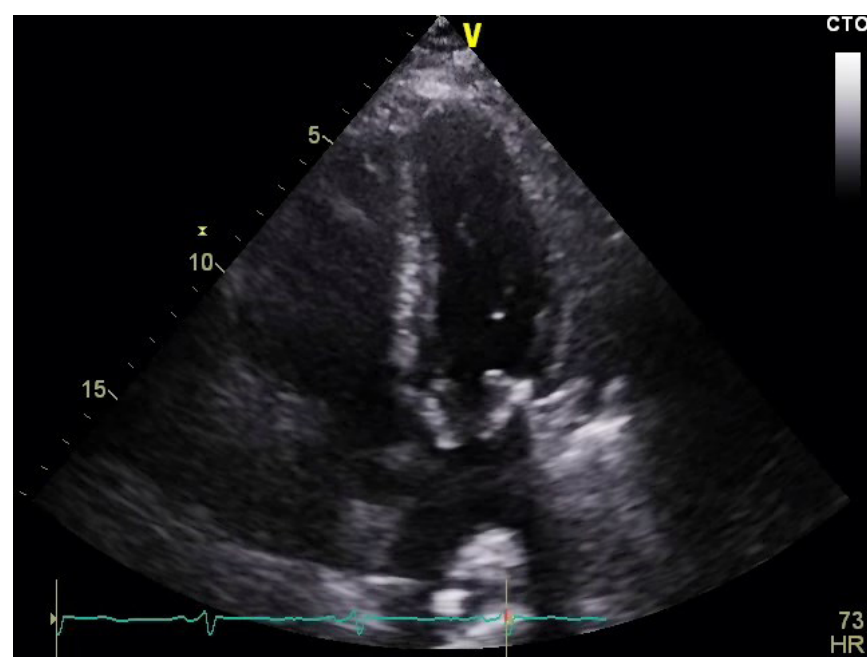

Figure 5. TTE, the apical four chamber view. Adequate position of the biological mitral valve.

atria, mostly in the left atrium, specificallyin approximately $75 \%$ of cases from the interatrial septum at the border of the fossa ovalis, and in $18 \%$ of cases from the right atrium [Keçeligil 1999; Lobo 2000]. Atrial myxoma is a rare benign cardiac tumor presenting with various signs and symptoms and the spectrum varies from completely asymptomatic to life-threatening events due to embolic phenomenon and obstruction of the mitral valve [Syed 2015]. The clinical manifestations usually depend on the anatomic position and size of the mass. There are 3 main types of presentations: embolic, obstructive, and constitutional. Embolic manifestations include visceral infarctions, stroke, and myocardial infarction. In a related study evaluating the risk factors for embolism in cardiac myxoma, location of the myxoma and irregular tumor surface were independently associated with increased risk of embolic complications [He 2015]. Localization is myxoma in both cases is atypical. In the first case, letf atrial myxoma concomitant with non Hodgkin's lymphoma and, without cardiovascular symptoms, which is described more in only one case, but in younger patients. Myxomas have been demostrated to produce numerous growth factors and cytokines [Oliveira 2010]. Studies have confirmed that cardiac myxomas produce interleukin-6 (IL-6) which is a multifactorial cytokine [Saji 1991; Medosa 2001]. It can be concluded that IL-6 has one of the key roles in the integration of non Hodgkin's lymphoma, and myxoma certainly stems from the production of IL-6. Surgical excision is the most effective treatment method, and provided fairly good outcomes in both of our cases. Echocardiography plays a prominent role in the diagnosis and follow-up of these patients. Transesophageal echocardiography provides very useful information, and is superior to transthoracic echocardiography in fully demonstrating the relationship between the tumor and the cavity wall, and also for the planning of surgery [Bruce 2011]. Our cases were followed by echocardiographies for a period 3 months and no recurrence of myxoma was identified during this period. 


\section{CONCLUSION}

The diagnosis of a cardiac myxoma is mostly done with echocardiography, both transthoracic and transesophageal, which represent the imaging modality of choice, although the latter permits precise information for the scheduling of surgery. Surgical excision is the most effective treatment method, and provided fairly good outcomes in both of our cases.

\section{REFERENCES}

Bruce CJ. 2011. Cardiac tumours: diagnosis and managment. Heart. 97:151-160.

He DK, Zhang YF, Liang Y, et al. 2015. Risk factors for embolism in cardiac myxoma: a retrospective analysis. Med Sci Monit 21:1146-1154.

Keçeligil HT, Demir Z, Kolbakır F, Demiră̆ MK, Akar H. 1999. Cardiac myxoma and surgical treatment. Türk Gögüs Kalp Damar Cer Derg 7:210-216.
Lobo A, Lewis JF, Conto R. 2000. Intracardiac masses detected by echocardiography: Case Presentation and Review of the Literature. Clin. Cardiol 23:702-708.

Medosa CE, Rosado MF, Bernal L. 2001. The role of Interleukin-6 in cases of Cardiac Myxoma: Clinical Features, Immunologic Abnormalities, and a Possible Role in Recurrence. Tex Heart Inst J 28:3-7.

Nath M P, Singh B, Chakrabarty A. 2011. Left atrial myxoma presenting as stroke-Case report and review of literature. The Indian Anaesthetists' Forum 1:1-6.

Oliveira R, Branco L, Galrinho A et al. 2010. Cardiac myxoma: a 13year experience in echocardiographic diagnosis. Rev Port Cardiol. 29:1087-1100.

Saji T, Yanagawa E, Matsuura H et al. 1991. Increased serum interleukin-6 in cardiac myxoma. Am Heart J 122:579-580.

Syed T, Ahir D, Gupta MK, Prasad A. 2015. Case Report of a Rare Benign Cardiac Tumor: Atrial Myxoma. Heart India 3:101-102. 